\title{
Grandes projetos urbanos: a apologia do distante e o receio do perto
}

\author{
ULTRAMARI, Clovis \\ CIFFONI, Ana Lucia
}

\section{Resumo}

Discute-se aqui a explicitação de entendimentos sobre a implantação de Grandes Projetos Urbanos (GPUs). Por gerarem impactos ostensivos na malha urbana, por reunirem grandes somas de recursos financeiros e por envolverem diversos agentes com interesses igualmente diversos, os GPUs são submetidos à constante análise social, econômica e política, além do escrutínio projetual de praxe. O artigo fundamenta-se em estudos dos seus autores sobre diferentes perspectivas assumidas perante os GPUs; aqui, os autores priorizam o entendimento de uma seleção da mídia impressa e contemporânea às obras de Haussmann, em Paris, da segunda metade do século XIX. O artigo parte de três pressupostos: (I) a tradicional crítica hoje observada em relação a essas grandes intervenções urbanas já era observada em momentos pretéritos, indicando assim um conjunto de características que lhes são intrínsecas e polêmicas, ainda que em geografias distintas; (II) o debate sobre GPUs é, por mais das vezes, extremado e não permite nuanças; e (III) na análise do observador distante, a apologia prevalece, talvez sugerida pelas qualidades da monumentalidade; já na análise daquele que lhe é próximo, persiste a crítica severa. O material utilizado foi uma seleção de artigos do jornal The New York Times que discutem as obras da chamada modernização de Paris, sob a responsabilidade de Haussmann. O debate e a conclusão se fundamentam sobre esse material e reiteram os pressupostos adotados.

Palavras-chave: Grandes Projetos Urbanos. Arquitetura Imagética. Gentrificação. Haussmann.

\begin{abstract}
:
This paper discusses the implementation of Large Urban Projects (LUPS). By generating clear impacts in the urban network, by assembling large amounts of financial resources, and by involving several agents with equally distinctive interests, LUPs are submitted to constant social, economical, and political scrutiny, as well as to routine project analysis. This paper is grounded on works by several researchers who regard LUPs from varying perspectives, all of which give priority to the understanding made public by print media that was contemporary to Haussmann's works in late nineteenth-century Paris. The present analysis is based on three main assumptions: 1) the traditional, current critique of LUPs has also been verified in the past, exposing a set of intrinsic and controversial features in these projects which can be found in different locations; 2) the LUP debate is often extremist and rejects conciliatory approaches; and 3) from the point of view of a distant observer, LUPs are generally regarded as positive, probably due to the monumentality appeal; from the point of view of a close observer, they are generally regarded as problematic. The material included articles from The New York Times which discuss Haussmann's works on the so-called modernization of Paris. The ensuing debate and conclusion are grounded on these sources and confirm the assumptions outlined.
\end{abstract}

Keywords: Large Urban Projects. Iconographic Architecture. Gentrification. Haussmann. 


\section{Dos debates sobre GPUs}

GPUs são entendidos como instrumentos de política pública, cuja escala projetual os distingue do entorno e impacta para além de seus limites imediatos. Identificados por interesses do capital privado, podem resultar de parcerias público-privadas e constituir uma das facetas mais presentes nos projetos estratégicos, seja quando de uma urgente (re)ocupação de áreas subtilizadas, seja quando da busca de uma refuncionalidade urbana requerida por novas e diversas realidades sociais ou econômicas. GPUs contam com uma multiplicidade de usos, porém, e majoritariamente, constituem estruturas para lazer, cultura, conservação do patrimônio, turismo, revitalização urbana e transporte (POWELL, 2000). $\mathrm{O}$ entendimento de Powell é reiterado por Del Rio (2000), para quem um GPU remete a oportunidades:

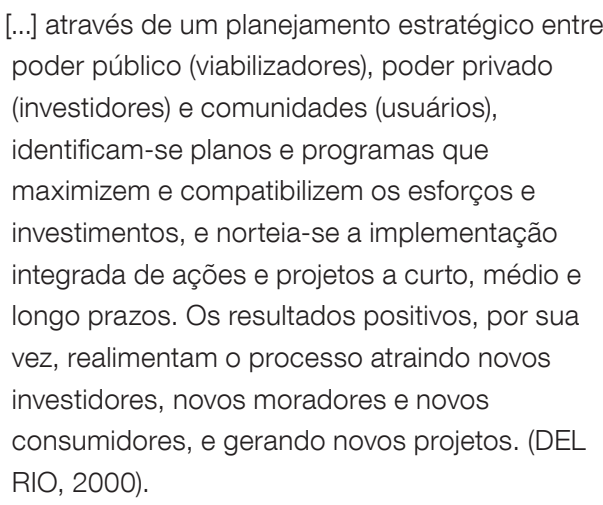

[...] através de um planejamento estratégico entre poder público (viabilizadores), poder privado (investidores) e comunidades (usuários), identificam-se planos e programas que maximizem e compatibilizem os esforços e investimentos, e norteia-se a implementação integrada de ações e projetos a curto, médio e longo prazos. Os resultados positivos, por sua vez, realimentam o processo atraindo novos investidores, novos moradores e novos consumidores, e gerando novos projetos. (DEL $\mathrm{RIO}, 2000)$.

Se Powell e Del Rio, dentre outros, formulam um conceito de Grande Projeto Urbano - sem de imediato destacar os interesses que tais intervenções suscitam -, outros autores adotam um entendimento mais dialético. Assim, os GPUs passam de uma intervenção capaz de alterar realidades para um processo quase estigmatizado como de interesse de uma minoria capaz de apropriar-se de seus resultados financeiros e elementos presentes no Planejamento Estratégico ou Projeto Cidade de Castells e Borja (1996). No lado da crítica mais contundente, evidenciam-se transformações da cidade por meio de projetos políticos e imobiliários que se valem de ícones arquitetônicos e urbanísticos facilmente identificados. Com isso, os GPUs constituem uma:

\footnotetext{
[...] espetacularização do urbano, uma certa encenação de uma vida pública que há muito deixou de existir... uma espécie de panacéia que por vezes não passa de recurso publicitário, quando não, inclusive, de inibição e controle cultural e social. (ARANTES, 1998, p. 25).
}

Harvey (2000, p. 144-145), em Spaces of hope, ao discutir o projeto de revitalização na cidade de Baltimore, serve-se de expressões como "the urban spectacle as a commodity" e "yuppie utopia", indicando forte e constante relação de gentrification e renewal. O mesmo autor, ao considerar as parcerias público-privadas, reitera os receios em relação aos GPUs: "every new wave of public investment is needed to make the last wave pay off. The private public partnership means that the public takes the risks and the private takes the profit" (HARVEY, 2000, p. 141).

O presente artigo procura identificar essas posições em diferentes tempos e espaços e reitera a ideia de extremos no entendimento do objeto que aqui se estuda. Em meio a receios e apologias, os GPUs resistem a marcar a história de muitas cidades, ora mais ora menos evidentes. No período pós-1980, em virtude das mudanças de padrões quando do anunciado - talvez precocemente - fim da cidade industrial, os GPUs se fizeram evidentes mais uma vez, levando alguns autores a caracterizar esse período - talvez também precocemente - de Renascença Urbana. Inicialmente circunscrita aos países ricos, como expressa Lungo (2002) e atualmente difundida entre países ditos emergentes (ULTRAMARI; FIRKOWISKI, 2012). Tal qual a Paris de Haussmann, a Viena do Imperador Franz Joseph e o Movimento City Beautiful de Burnham, os GPUs contemporâneos são criticados especialmente por causa do processo de gentrification que possam gerar, à eventual malversação de recurso público, à possível apropriação política de sua imagem, aos ganhos imobiliários de grupos envolvidos, à expulsão de população original e muitas vezes a uma inapropriada apologia de um passado que talvez nunca tenha existido. ${ }^{1}$ Nesta postura receosa, há uma evidente minimização de eventuais aspectos positivos, tais como a revalorização ou a refuncionalização de áreas subutilizadas, a otimização de infraestruturas disponíveis, a disponibilização de espaços de lazer e cultura, o incremento de atividades turísticas e, em especial, a recuperação de autoestima cidadã.

GPUs expressam uma nova dinâmica da sociedade, sobretudo observada no final dos anos 1970 e início dos 1980 (ULTRAMARI, 2005), que envolve uma forte frustração à gestão urbana, de modo geral, e à sua incapacidade financeira, técnica e política para implementar intervenções socioeconômicas há muito desejadas. Neste mesmo período, a cidade assume novas funções, agora terciárias, em espaços tornados obsoletos pela reconversão econômica ou pela reespacialização da indústria. Na reconstrução

1. Refere-se aqui à chamada "museificação" de espaços urbanos quando de projetos de revitalização. 
desses espaços, envolvendo grandes oportunidades e também grandes recursos, e quase sempre fundamentada em GPUs, interesses diversos se concretizam e se agudizam (VALE; CAMPANELLA, 2005). Em países de urbanização mais consolidada e com maiores disponibilidades de recursos para responder a exigências de ineditismos arquitetônicos e tecnológico-construtivos que caracterizam os GPUs, pode-se concluir que esses tenham mesmo anunciado uma possível revolução no interior de suas cidades (LUNGO, 2000; LUNGO; SMOLKA, 2005). Em outras realidades, aceitar esse fenômeno tal qual requer grandes dúvidas.

No debate sobre GPUs, estes quase sempre são vistos apenas por uma de suas vertentes: ou a resposta urbanística da sua arquitetura e suas características ambientais, ou a vertente política de oportunidades de aplicação de recursos públicos nesta ou naquela ação. Perde-se a oportunidade de entender o resultado do emprego de capital público com uma determinada feição muito própria de cada momento por que passa a sociedade. Esta reflexão fica mais clara ao se analisar, no item a seguir, o projeto de Haussmann, onde se traz, à luz de um novo tempo, o debate que lhe foi contemporâneo. Perdese, também, a oportunidade de se discutir a própria cidade, ou seja, sobre a eventual capacidade de eles alavancarem mudanças para além de suas áreas de impacto mais imediato. Em meio a potenciais não apropriados, a prioridades pouco discutidas e a formas de gestão não atualizadas, os GPUs são vistos com desencanto, crítica e, apenas eventualmente, com alguma apologia. Reconhece-se, no entanto, que resta melhor explorar: os seus propósitos, o seu potencial em agregar a sociedade no debate de suas cidades, as suas oportunidades integradoras para projetos estratégicos, os seus impactos na paisagem, bem como o correto uso do recurso público em sua implantação.

\section{O estudo}

A fonte de pesquisa utilizada são artigos disponibilizados on-line pelo jornal The New York Times (TNYT). A seleção inicial foi feita com a palavra-chave "Haussmann", iniciando em 1858 (começo da disponibilização de arquivos) e finalizando em 1900, acreditando-se ser esse o limite temporal para uma análise contemporânea das referidas intervenções parisienses. ${ }^{2}$ O refinamento da pesquisa reviu o corte para 18681896, resultando em quinze artigos, além do conhecido obituário de Haussmann.

Quadro 1: Artigos selecionados para a pesquisa

\begin{tabular}{|l|l|}
\hline TíTULO & PUBLICAÇÃO \\
\hline The French capital: Paris rebuilt, ... Asphaltum versus macadam & $13 / 12 / 1867$ \\
\hline The French capital: moral aspect of the city / Baron Haussmann's improvement upon it & $09 / 05 / 1868$ \\
\hline The French capital: the crusade against Baron Haussmann & $05 / 07 / 1868$ \\
\hline Baron Haussmann: his fabulous wealth & $01 / 02 / 1870$ \\
\hline Haussmann and Hall & $17 / 07 / 1871$ \\
\hline The spell of Paris & $12 / 05 / 1874$ \\
\hline Jules Ferry & $05 / 04 / 1885$ \\
\hline Haussmann and his boulevard & $25 / 12 / 1888$ \\
\hline Haussmann's boulevard: steps to complete it. What it will cost & $18 / 03 / 1890$ \\
\hline Baron of Haussmann. Mémoirs du Baron Haussmann & $11 / 05 / 1890$ \\
\hline Baron Haussmann's work & $20 / 07 / 1890$ \\
\hline Paris Haussmannised & $18 / 08 / 1890$ \\
\hline Haussmann and his work: before the empire and after & $26 / 01 / 1891$ \\
\hline Paris dispatch to the London Daily Telegraph (nota) & $01 / 02 / 1891$ \\
\hline London needs a Haussmann & $07 / 07 / 1896$ \\
\hline Haussmann's obituary & $11 / 01 / 1891$ \\
\hline
\end{tabular}

Fonte: The New York Times

2. Haussmann falece com 81 anos, em 11 de janeiro de 1891, sendo prefeito do Departamento do Sena, de 1853 a 1870. 
A primeira observação que se pode fazer da leitura dos artigos selecionados é a recorrência do tratamento da questão das reformas de Paris, sobretudo na década de 1860, pela imprensa estrangeira selecionada para o presente estudo. Outras pesquisas poderiam confirmar que a visão recorrentemente positiva encontrada na grande maioria dos artigos aqui estudados não se repete na imprensa parisiense, mais familiarizada com questões diversas dos projetos haussmaniannos. A partir de artigo no TNYT que relata o lançamento das memórias do prefeito agora já aposentado, pode-se construir uma síntese de como o responsável pelas obras é entendido por seus conterrâneos:

[...] in Russia, everybody is a Nihilist except the Czar; in Paris everybody was against Haussmann - capitalists, merchants, laborers, those who liked the government and feared for his apparent heedlessness, those who hated the Emperor and regarded the Prefect as his "âme damnée" even the emperor, who approved his plans, signed the decrees that he wanted, intervened in the personal quarrels that blocked his way but insisted publicly that taxes on the necessities should be reduced. (PARIS HAUSSMANNISED, 18 ago. 1890).

Essa visão crítica é, todavia, ignorada quando Paris é relatada tão somente como uma cidade estrangeira submetida a reformas de grande vulto, embelezadoras e saneadoras: enaltecem-se o acréscimo de vias, suas caixas mais largas, sua iluminação mais intensa, sua oferta de água mais abrangente, suas árvores valorizando paisagens, dentre outros. Ao mesmo temo, diminui-se a importância, consciente ou não, das relocações impostas aos moradores das áreas em obras e dos valores das indenizações pagas a eles. Para a mídia estrangeira tudo parecia acontecer sem crítica, nem mesmo em relação a um homem e a um projeto urbanístico que "never sacrificed a perspective to time or money" (PARIS HAUSSMANNISED, 18 ago. 1890). Mesmo no caso das críticas mais conhecidas, como a da malversação dos recursos públicos, a postura adotada é da exclusão explícita do debate desse possível delito: "He [Haussmann] was capable, fearless, and devoted to the Emperor" e por isso os parisienses lhe deveriam ser gratos, pois o "tirano" não fez fortuna, mas sim a perdeu (PARIS HAUSSMANNISED, 18 ago. 1890).

The widest streets of their Paris were the rue Saint Honoré and the rue Saint Antoine: the Champs Elysées was a desert or a swamp; the Bois de Boulogne was at the other end of the world. Water from the Seine or the Ourcq Canal was warm in Summer, cold in Winter, was hawked in the streets from water carts by Auvergnats. There were not enough reservoirs to clean the streets. The sewer system was wretched. In 1852 there were 384 kilometers of streets, avenues and boulevards, averaging 12 meters in width: in 1869, 845 averaging 12 meters in width. The suppressed streets and alleys averaged 7 meters in width. There were in 1852287 kilometers of sidewalks having a surface of 73 hectares; in 1869, 1.088 with a surface of 296. There were 50.400 trees in 1852 , 95.577 in 1869. There were 12.400 gas and 85 lamp lights in 1852, 32.300 gas and 1.539 lamp lights in 1869. There were demolished in seventeen years 19.722 houses; built and rebuilt 43.777. There were dislodged 117.553 families.

Essa mesma visão utilitarista que prioriza o fim que não analisa os meios toma a justificativa maior das obras de Haussmann, a militar, como inerente a uma intervenção urbanística. De fato, a estreita relação do projeto de Haussmann com o desejo de pôr fim a movimentos sociais ou mesmo à "vitória" desse urbanismo sobre a Comuna de Paris ${ }^{3}$, em 1871, va sans dire nos artigos selecionados, propensos a uma seleção de fatos menos questionáveis.

\footnotetext{
When the Empress said to him, looking at one of his long, straight boulevards just opened, "Why did you make that Boulevard so long and straight, it is tiresome?" "Madame", replied the Prefect, "I made it very long and very straight because the Generals of artillery whom I consulted a great deal on the subject, assured me that it was impossible to teach cannon balls to turn round the first corner to the left". (THE FRENCH CAPITAL, 13 dez. 1867).
}

Do mesmo modo que se observava sem criticar o suporte militar que as obras de Haussmann aportavam, parecia haver uma aprovação generalizada de que o urbanismo podia, além de garantir essa estratégia militar, também regular a moral do cidadão. De fato, a mesma valorização, velada ou não, da limpeza da paisagem urbana capaz de permitir fluidez e segurança, parecia também coibir imoralidades:

\footnotetext{
[...] a man will do in a narrow, dark and dirty street what he will not do in a wide, open, clean street. It is exactly the difference between night and day. Handsome and clean streets bring well-dressed people, by the law of imitation;
}

3. A Comuna de Paris é um poder instalado por dois meses em Paris, em 1871, vinculado a descontentamentos da classe trabalhadora e à derrota da França diante da Prússia. 
communicate their habits to those about them. (THE FRENCH CAPITAL, 13 dez. 1867).

A mesma relação acima observada entre uma moral enaltecida e facilitada por meio de um modelo de urbanismo saneador pode também ser encontrada em artigo publicado posteriormente:

[...] not long ago I made use of the expression, for example, that Paris was the most moral-looking city in the world. Of course I could not venture any stronger expression than that [...] but a great deal has been gained when the streets and public spaces have been purified; the mysterious under strata is then in turn more easily attacked. (THE FRENCH CAPITAL, 9 maio 1868).

Nas limitações deste estudo que aqui busca similaridades entre análises do passado e análises contemporâneas a respeito de Grandes Projetos Urbanos, chama a atenção o uso do chamado "interesse da maioria" ou "interesse público" para justificar as intervenções, ainda que tais interesses sejam de difícil delimitação e que possam eventualmente explicar desapropriações. Nos artigos do TNYT, a visão de apologia dirigida às obras de Haussmann obriga seus autores a passar ao largo das tradicionais e conhecidas disputas presentes em processos fundiários.

\footnotetext{
This word "public utility" is the word put forward in all these plans of improvement; for who can object to what is for the public good? When at last a new street or public square is decided on by the prefect and his legal adviser, the city engineer ad his aids survey the route, the people living thereon are notified that at a certain date they must evacuate the premises they occupy (two year's notice is generally given). (THE FRENCH CAPITAL, 13 dez. 1867).
}

O olhar estrangeiro - propositado, velado, ou desconhecidamente pouco sensível a questões que implicam nos interesses da população local de Paris - prossegue em diferentes momentos que a seleção dos artigos permite discutir. Assim, mesmo com a queda de Napoleão III,4 em 1870, a crise financeira que se segue, e mesmo as ideias difundidas pela Comuna de Paris, as obras de Haussmann - estritamente vinculadas a um poder derrubado - conseguem manter o apelo na mídia internacional analisada. Tais obras persistem em serem vistas tão somente como um interminável espetáculo de grande escala e de novidades, dificilmente se propondo a questionar "problemas menores locais". Assim,

4. Charles-Louis-Napoléon Bonaparte, presidente eleito por voto em 1848, após golpe de 1851, assume como Napoleon III em 1852. Em 1870, é capturado. ainda em 1874, quatro anos após o fim do poder dado a Haussmann, a apologia se mantém.

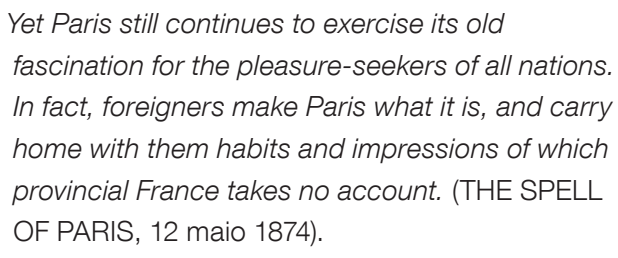

Esse olhar estrangeiro, capaz de filtrar o que the interessa e o que lhe pode afetar, transforma Paris em modelo a ser seguido, da mesma maneira que hoje muitas cidades perseguem a reprodução de um sucesso experimentado pelas transformações em Barcelona ou Bilbao a partir de Grandes Projetos Urbanos. Essa visão de cidade idealizada transparece em artigo selecionado de 1896, muito depois do final da era Haussmann:

\section{"London needs a Haussmann: what London really wants is a Baron Haussmann to transform the metropolis as Paris was metamorphosed by the cutting of grand boulevards through slums" (LONDON NEEDS A HAUSSMANN, 7 jul. 1896).}

O que se observa nas preferências dos temas discutidos nos artigos apresentados é, pois, a perspectiva de alguém que busca se apropriar dos resultados, apenas. Com isso, do mesmo modo como as cidades que implementam Grandes Projetos Urbanos na atualidade, Paris parece ser produzida para um novo morador, não mais para sua população original. Paris é, então, recorrentemente apresentada nos artigos selecionados como uma cidade que "merece ser visitada", indicando que suas obras atendem aos interesses de outrem que não necessariamente de sua população local:

\footnotetext{
"[...] families must be able to visit Paris for weeks and months, to see its grand palaces and museums and other feeling than that of pleasure. There must be no disagreeable souvenir of any kind" (THE FRENCH CAPITAL, 9 maio 1868).
}

Por último, outra constante observada na leitura dos artigos selecionados é, do mesmo modo que na análise de Grandes Projetos Urbanos contemporâneos, uma recorrente conexão entre essas obras e seu uso pelo poder político instalado e pelo capital privado parceiro. Se a seleção de artigos se distrai na discussão sobre as características tão somente arquitetônicas e urbanísticas dos resultados, Haussmann nos confirma o que já se sabia na discussão de projetos contemporâneos. Assim, um artigo selecionado que discute as memórias de Haussmann ${ }^{5}$ reproduz algumas de suas ideias, dentre as quais é 
evidenciada a subserviência de suas ações a um projeto político de estado:

\author{
[...] where, asks HAUSSMANN, with evident \\ elation, would the troops of Versailles have been \\ but for the great lines of boulevards which he \\ traced through the crowded quartiers of the \\ working classes? [...] HAUSSMANN first \\ pampered the artisans of the capital with \\ abundance of work artificially created, and then \\ disgusted them with the hardness of existence in \\ the Paris for the rich alone, which he had \\ created. (HAUSSMANN AND HALL, 17 jul. 1871).
}

Da fonte com que se trabalhou neste item é possível arriscar caracterizá-la como distante das questões locais, atenta mais aos resultados do que aos meios utilizados para a implementação das transformações. Ao longe da realidade, os artigos do TNYT sobre a Haussmann em Paris seguem sem relatar impactos negativos, sem externalidades e sem disputa entre os inúmeros agentes que uma intervenção nessa escala sempre agrega. Comprova essa conclusão a observação de que as únicas pessoas capazes de consistentemente questionar as obras de Haussmann são os entusiastas da preservação de velhos edifícios, ainda que sem observar aspectos de saúde e conveniência públicas (HAUSSMANN AND HIS BOULEVARD, $25 \mathrm{dez}$. 1888). Nesta visão linear, o mesmo artigo finaliza afirmando que:

\section{[...] they [os críticos às intervenções em Paris] should honor Baron Haussmann as a distinctly modern man, who did much to obliterate the traces of the past by doing away with tortuous, narrow streets and the old houses that linked the present to the times when kings were everything in France and Municipal councilors nothing. (HAUSSMANN AND HIS BOULEVARD, 25 dez. 1888).}

Certamente, a seleção de fontes aqui utilizada (um jornal apenas) recomenda cautela nas conclusões deste item. Ampliar essa fonte de informação estrangeira e confrontá-la com outra lo$\mathrm{cal}^{6}$ poderia confirmar uma das hipóteses aqui lançadas, que é a de uma distinção no olhar de quem analisa o fenômeno em questão, segundo maior ou menor proximidade ou distanciamento. Todavia é possível confirmar a permanência

\footnotetext{
5. Haussmann dedica seus três últimos anos de vida a escrever suas memórias, cuja publicação é motivo, por mais de uma vez, de artigo no jornal The New York Times da época.

6. Ensaiou-se a mesma pesquisa junto ao Jornal Le Monde, porém esse não conta com a mesma disponibilidade on-line que o TNYT; os seus arquivos estão disponíveis apenas a partir de 1983.
}

temporal de determinadas questões na análise de um Grande Projeto Urbano. Estão presentes nos artigos selecionados, ainda que apresentadas de forma explicitamente parcial, questões referentes a: interesses de Estado no uso da arquitetura e do urbanismo, disputa entre o antigo e o novo em processos de demolição e relocação, reposicionamento hierárquico da cidade em um cenário internacional (a partir de intervenções imagéticas), generalizações conceituais estratégicas (no entendimento do que se pode considerar "interesse público"), e adequação do espaço urbano para uso por outrem, externo à história da cidade.

\section{Considerações finais}

A leitura dos documentos levantados - ainda que geográfica e temporalmente distantes de nossa contemporaneidade - sugere uma característica intrínseca das grandes intervenções urbanas que se concretiza na polêmica, no receio e na crítica. Todavia, se GPUs provocam receios e críticas, também aportam apologias exageradas e visões parciais. A razão de tais receios e críticas estaria na intervenção que lhes é inerente: alteram histórias, alteram paisagens conhecidas, utilizam grandes volumes de recursos, sugerem novas e talvez arriscadas parcerias, alertam para o debate sobre prioridades urbanas. Enfim, contam com potencial para desestabilizar realidades urbanas há muito consolidadas. Na razão do apoio irrestrito, poderia estar o distanciamento do fato analisado, um reduzido conhecimento das necessidades da população diretamente envolvida e uma possibilidade de ser, tão somente, um usuário estrangeiro dos resultados.

Em relação ao debate que um GPU possa gerar, vale lembrar que o projeto arquitetônico e a função a ser dada por ele ofertam as bases de uma discussão fora do comum entre nós: a da crítica arquitetônica. Porém, estaríamos ainda distantes da convicção de que a obra arquitetônica, qualquer uma, está inserida num contexto urbano que é público e que afeta a todos, ampliando o universo de interessados num debate sobre sua função e sua localização na cidade, dentre outros. A construção de GPUs permite, então, uma situação pouco habitual e que inclui polêmicas sobre aspectos diversos, que vão além da simples preocupação com o recurso público e a crítica político-ideológica, mas que pode avançar para a crítica arquitetônica propriamente dita e prosseguir para seus impactos urbanísticos.

O que chama a especial atenção na leitura da fonte selecionada é que a crítica e a análise dos GPUs dificilmente potencializam o debate sobre 
a cidade como um todo. Na discussão daquilo que porventura pode ser o novo, a permanência que se tem é a da tradição interpretativa da cidade. Tem-se, pois, que a limitação analítica de um GPU é o seu vínculo com a definição de prioridades para o coletivo da cidade. Demarcar o debate em tempo posterior a essa definição poderia revelar, talvez, uma possível opção entre se edificar museus e a urgência de obras para usos emergenciais, tais como segurança, saúde ou educação (todos, tradicionais exemplos de GPUs contemporâneos). Quando da difícil desvinculação de um GPU da discussão sobre as prioridades de uma cidade, arrisca-se não avançar para outros níveis de propostas estratégicas; quando da existência do debate, arrisca-se não ganhar com possíveis resultados positivos desse mesmo GPU. Ganha-se, porém, na saudável discussão sobre "as coisas da cidade".

\section{Referências}

ARANTES, O. Urbanismo em fim de linha: e outros estudos sobre o colapso da modernização arquitetônica. São Paulo: Edusp, 1998.

BARON Haussmann: his fabulous wealth. The New York Times, 1 fev. 1870. Disponível em:<http://query.nytimes.com/gst/abstract.html ?res=9507E6D6103BE63BBC4953DFB466838B 669FDE>. Acesso em: 15 dez. 2012.

BARON of Haussmann.Mémoirs du Baron Haussmann.The New York Times, 11 maio 1890. Disponível em: <http://query.nytimes.com/gst/ abstract.html?res=9A07E3DD123BE533A25752 C1A9639C94619ED7CF>. Acesso em: $15 \mathrm{dez}$. 2012.

BARON Haussmann's work. The New York Times, 20 jul. 1890. Disponível em: <http://query. nytimes.com $/ \mathrm{mem} /$ archive-free/pdf?res=9F01E6 DF1239E033A25753C2A9619C94619ED7CF>. Acesso em: 15 dez. 2012.

CASTELLS, M.; BORJA, J. As cidades como atores políticos. Novos Estudos, n. 45, p. 152-156, 1996.

DEL RIO, V. Em busca do tempo perdido: o renascimento dos centros urbanos. Vitruvius. Disponível em:<http://www.vitruvius.com.br/revistas/read/arquitextos/01.006/963>. Acesso em: 2 dez. 2009.

HARVEY, D. Spaces of hope. Berkeley: University of California Press, 2000.
LUNGO, M. Macroproyectos urbanos: desafíos para las ciudades latinoamericanas. Land Lines, Lincoln Institute of Land Policy, v. 14, n. 4, 2000.

LUNGO, M.; SMOLKA, M. O. Land value and large urban projects: the Latin American experience. Land Lines, Lincoln Institute of Land Policy, v. 17, n. $1,2005$.

HAUSSMANN and Hall. The New York Times, 17 jul. 1871. Disponível em: <http://query.nytimes. com/gst/abstract.html?res=9900E4D7103EEE34 BC4F52DFB166838A669FDE>. Acesso em: 15 dez. 2012.

HAUSSMANN and his boulevard. The New York Times, 25 dez.1888. Disponível em:<http://query. nytimes.com/gst/abstract.html?res=9E0CEODD 163BE033A25756C2A9649D94699FD7CF>.

Acesso em: 15 dez. 2012.

HAUSSMANN and his work: before the empire and after. The New York Times, 26 jan. 1891. Disponível em: <http://query.nytimes.com/gst/ abstract.html?res=9E0DE2DD1F3BE533A25755 C2A9679C94609ED7CF>. Acesso em: $15 \mathrm{dez}$. 2012.

HAUSSMANN'S boulevard: steps to complete it. What it will cost. The New York Times, 18 mar. 1890. Disponível em: <http://query.nytimes.com/ gst/abstract.html?res=9D0DE3DB153BE533A25 75BC1A9659C94619ED7CF>. Acesso em: 15 dez. 2012.

JULES F. The New York Times, 5 abr. 1885. Disponível em: <http://query.nytimes.com/mem/ archive-free/pdf?res=FB0B10FD3A5C15738DD DAC0894DC405B8584F0D3>. Acesso em: 15 dez. 2012.

LONDON needs a Haussmann. The New York Times, 7 jul. 1896. Disponível em: <http://query. nytimes.com/gst/abstract.html?res=9D02E6D61 730E033A25754C0A9619C94679ED7CF>.

Acesso em: 15 dez. 2012.

PARIS Haussmannised. The New York Times, 18 ago. 1890. Disponível em: <http://query.nytimes. com/gst/abstract.html?res=9903E6DE1239E033 A2575BC1A96E9C94619ED7CF>. Acesso em: 15 dez. 2012.

PARIS dispatch to the London Daily Telegraph (nota). The New York Times, 1 fev. 1891. Disponível em: <http://query.nytimes.com/gst/ abstract.html?res=9C00E2DC1F3BE533A25752 C0A9649C94609ED7CF>. Acesso em: $15 \mathrm{dez}$. 2012. 
POWELL, K. City transformed: urban architecture at the beginning of the 21st century. New York: Te Neues, 2000.

THE FRENCH capital: Paris rebuilt, the process of transformation, French mode of expropriation, the city streets, Asphaltum versus macadam. The New York Times, 13 dez. 1867. Disponível em: <http://query.nytimes.com/gst/abstract.htm I?res=9F00EFD6163BE63BBC4B52DFB467838 C679FDE>. Acesso em: 4 jan. 2011.

THE FRENCH capital: moral aspect of the city / Baron Haussmann's improvement upon it. The New York Times, 9 maio 1868. Disponível em:<http://query.nytimes.com/gst/abstract.html ?res=9D04E2DA1730EE34BC4153DFB3668383 679FDE>. Acesso em: $15 \mathrm{dez} .2012$.

THE FRENCH capital: the crusade against Baron Haussmann. The New York Times, 5 jul. 1868. Disponível em: <http://query.nytimes.com/gst/ abstract.html?res=9802E0DD1E3CE13BBC4D53 DFB1668383679FDE>. Acesso em: 15 dez. 2012.

THE SPELL of Paris. The New York Times, 12 maio, 1874. Disponível em: <http://query.nytimes. com/gst/abstract.html?res=9C06E4DF143BEF3 4BC4A52DFB366838F669FDE $>$. Acesso em: 15 dez. 2012.

ULTRAMARI, C. O fim das utopias urbanas. São Paulo: Nobel, 2005.

ULTRAMARI, C.; FIRKOWISKI, O. L. C de F. Sobre mudanças e continuidades na Gestão Urbana Brasileira. Mercator, Fortaleza, v. 11, n. 24, p. 73-88, 2012.

VALE, L. J.; CAMPANELLA, T. J. The resilient city: how modern cities recover from disaster. New York: Oxford University Press, 2005. 